AN MED INTERNA (Madrid) Vol. 20, N. ${ }^{\circ}$, pp. 399-402, 2003

\title{
Intoxicaciones digitálicas sospechadas y confirmadas
}

\author{
P. MÁIQUEZ ASUERO, J. A. ABADÍN DELGADO, C. JIMÉNEZ PLATA, \\ A. SÁNCHEZ ROMERO ${ }^{1}$, J. A. DURÁN QUINTANA
}

Hospital Universitario Virgen Macarena. Sevilla.

${ }^{1}$ Complejo Hospitatario de Albacete. Albacete.

CONFIRMED AND SUSPECTED DIGOXIN INTOXICATIONS

\section{RESUMEN}

Objetivos: Cuantificar y analizar loas intoxicaciones digitálicas y sus sospechas.

Método: Se ha realizado un estudio de utilización de medicamentos sobre las peticiones de digoxinemia y los resultados obtenidos en pacientes tratados crónicamente con digoxina.

Resultados: Se sospechó intoxicación digitálica (presencia de síntomas y signos propios de la misma) en el $31,3 \%$ de los pacientes y se confirmó analíticamente (digoxinemia $>2 \mathrm{ng} / \mathrm{mL}$ ) en el 16,6\%. El sexo, la edad y la dosis no influyeron significativamente en la sospecha ni en la confirmación de la intoxicación digitálica. Sí influyeron en las sospechas de intoxicación digitálica, la hospitalización y la asociación de fármacos hipokalemiantes o que eleven la digoxinemia. En las intoxicaciones confirmadas analíticamente no se encontraron diferencias significativas entre las sospechadas y las no sospechadas.

Conclusiones: La sospecha de esta intoxicación no suele relacionarse con los niveles plasmáticos de digoxina y, por tanto, su límite tóxico es impreciso.

PALABRAS CLAVE: Intoxicación digitálica. Digoxinemia. Geriatría.

\begin{abstract}
Aims: To quantify and analyze suspected and actual digoxin intoxications.

Method: A drug-use study has been carried out of digoxinaemia requests and results in patients treated chronically with digoxin.

Results: Digoxin intoxication (presence of symptoms and typical signs) was suspected in $31.3 \%$ of the patients. The percentage analytically confirmed intoxications (digoxinaemia $>2 \mathrm{ng} / \mathrm{mL}$ ) was $16.6 \%$. Sex, age, and dosage had no significant effect in the suspicion or confirmation of digoxin intoxication. Hospitalization and the association of hypokalaemic drugs or those increasing digoxinaemia had no effect in the suspicion of digoxin intoxication. In analytically confirmed intoxications, no significant differences were found between suspected and non-suspected cases.

Conclusions: The suspicion of this intoxications is not usually related with digoxin serum levels, and thus, the toxic limit is imprecise.
\end{abstract}

KEY WORDS: Digoxin toxicity. Elderly patients. Serum levels.

Máiquez Asuero P, Abadín Delgado JA, Jiménez Plata C, Sánchez Romero A, Durán Quintana JA. Intoxicaciones digitálicas sospechadas y confirmadas. An Med Interna (Madrid) 2003; 20: 399-402.

\section{INTRODUCCIÓN}

El problema más común de la terapéutica con digoxina sigue siendo el riesgo de intoxicación con dicho fármaco. La incidencia de tal intoxicación se ha reducido en los últimos años, tanto en los pacientes hospitalizados como en los ambulatorios (1-5).

El motivo principal de esta reducción es la determinación habitual de la digoxinemia; su petición se efectúa con frecuencia sin especificar el motivo $(3,6)$. Por el contrario, hay sospechas de intoxicación digitálica en las que no se encuentran niveles tóxicos de digoxina (7). Por otro lado, es relativamente frecuente el hallazgo casual de una digoxinemia tóxica sin sintomatología (3,4,8-10). En consecuencia, existen sospechas de intoxicación digitálica que no se confirman analíticamente, e intoxicaciones por digoxina que no se sospechan (11-13).

Los objetivos del presente trabajo son cuantificar y analizar las intoxicaciones digitálicas y sus sospechas en la población estudiada.

\section{MATERIAL Y MÉTODO}

Se ha realizado un estudio de utilización de medicamentos, transversal, retrospectivo, durante el periodo mayo 2000-abril

Trabajo aceptado: 4 de febrero de 2003

Correspondencia: Pilar Máiquez Asuero. Departamento de Farmacología, Pediatría y Radiología. Facultad de Medicina. Avda. Sánchez Pizjuán, 4. 41009 Sevilla. e-mail: jaduran@us.es. 
2001, sobre las peticiones de niveles plasmáticos de digoxina recibidas en el Servicio de Farmacología Clínica del Hospital Universitario Virgen Macarena (Sevilla, España). Se incluyeron todas las peticiones de los pacientes en tratamiento estable con digoxina por vía oral ( $\geq 7$ días) y con edad $\geq 60$ años. De las peticiones se recogieron los siguientes factores determinantes: sexo, edad, procedencia de la petición, síntomas descritos, posología de la digoxina, fármacos asociados y patologías concomitantes (Tabla I). Las determinaciones de digoxina se efectuaron mediante inmunoanálisis de polarización fluorescente.

\section{TABLA I}

FACTO RES DETERM INANTES RECO GIDOS DE LAS HOJAS DE PETICIÓ N DE NIVELES SÉRICOS DE DIGOXINA

Sexo: hombre, mujer.

Grupos de edad: 60-70, 71-80, >80 años.

Procedencia de la petición: ambulatoria, hospitalaria.

Síntomas descritos: ver Tabla II.

Grupos de dosis: 250 mg/ día, 250 mg/ día excepto sábados y domingos, $125 \mathrm{mg} /$ día, 125 $\mathrm{mg} /$ día excepto sábados y domingos.

Fármacos asociados: los que producen hipopotasemia, las que elevan la digoxinemia

Las variables principales fueron la sospecha de intoxicación digitálica (SI/NO) y la confirmación analítica de la intoxicación (SI/NO). Se consideró sospecha cuando constaba así en la hoja de petición de la determinación de la digoxinemia, o cuando existía 1 o más de los síntomas sugestivos de intoxicación digitálica (Tabla II); de no ser así, se consideraba que no había sospecha de intoxicación. Se consideró intoxicación digitálica cuando el nivel plasmático de digoxina era $>2$ $\mathrm{ng} / \mathrm{mL}$ (La mayoría de los autores aceptan que el límite tóxico de la digoxina es $>1,8 \mathrm{ng} / \mathrm{mL}$ (14)). Estas variables se distribuyeron y compararon según los factores determinantes antes citados, recogidos de la hoja de petición.

\section{TABLA II}

\section{CRITERIOS DE SO SPECHA DE INTO XICACIÓ N DIGITÁLICA}

Síntomas digestivos: Náuseas, vómitos, diarrea, dolor abdominal, anorexia

Síntomas neurológicos: Síndrome vertiginoso, mareos, temblor generalizado, inquietud

Síntomas generales: $\quad$ M alestar general, astenia, cuadro vegetativo, hipotensión, hipopotasemia, síncope

$\begin{array}{ll}\text { Síntomas } & \text { Bradicardia, taquicardia, fibrila- } \\ \text { electrocardiográficos: } & \text { ción auricular con respuesta } \\ & \text { ventricular lenta, ekg sospecho- } \\ & \text { so, extrasístoles supraventricula- } \\ & \text { res, bigeminismo, arritmia ven- } \\ & \text { tricular, cubeta digitálica, } \\ & \text { "trastorno de ritmo" }\end{array}$

Como el trabajo no es un ensayo clínico sólo se pidió a los pacientes autorización para usar de forma anónima los datos de sus historias clínicas respectivas.

Se efectuó una búsqueda bibliográfica en Medline ${ }^{\circledR}$ (1.980-2.001), utilizando como palabras clave: digoxin toxicity, elderly patients y serum levels.

Los datos fueron analizados mediante el paquete estadístico SPSS (versión 8,0). Las variables principales se describieron mediante su proporción. Se realizaron comparaciones empleando la prueba de Chi cuadrado y la asociación entre las variables principales se analizó mediante su "odds ratio" (OR). La significación estadística se fijó en p $<0,05$.

\section{RESULTADOS}

En el periodo de estudio se recibieron 786 peticiones de niveles séricos de digoxina, de las que solo 403 (51,3\%) tenían $\geq 60$ años y estaban en tratamiento con digoxina $\geq 7$ días. Esta población de estudio (231 mujeres, 172 hombres) presentaba una edad media de 73,4 $\pm 8,0$ años.

Se sospechó intoxicación digitálica en la tercera parte (126; 31,3\%) (Tabla III). En 80 de ellos existía un solo síntoma sospechoso de intoxicación digitálica. Los síntomas más frecuentes fueron los digestivos (90) y los electrocardiográficos (72). El número de pacientes intoxicados (concentraciones séricas de digoxina $>2 \mathrm{ng} / \mathrm{mL}$ ) fue reducido (67: 16,6\%).

En los pacientes que se sospechó intoxicación digitálica sólo se confirmó en 28/126 (22,2\%). En las intoxicaciones confirmadas sólo se sospechó en 28/67 (41,8\%). La probabilidad de sospecha de intoxicación fue mayor, pero no significativa, en los pacientes intoxicados que en los que no lo estaban (OR=1,74; IC 95\%: 0,98-3,09) (Tabla III).

El sexo, los grupos de edad y los grupos de dosis concomitantes no influyeron significativamente en la sospecha de intoxicación digitálica. Por el contrario, sí se encontró con la procedencia de la petición, siendo la proporción de sospecha o no de intoxicación digitálica significativamente mayor en los pacientes hospitalizados que en los ambulatorios (Tabla IV). También se encontraron diferencias significativas entre las sospechas de intoxicación digitálica y la asociación de fármacos hipokalemiantes (Tabla V) o que eleven la digoxinemia (Tabla VI).

En relación a la confirmación de intoxicación digitálica, no se encontraron diferencias significativas en cuanto al sexo, los grupos de edad, la procedencia de la petición, los grupos de dosis y los fármacos asociados (hipokalemiantes o que eleven la digoxinemia).

\section{TABLA III}

RELACIÓ N ENTRE SO SPECHA E INTO XICACIÓ N DIGITÁLICA

\begin{tabular}{lcrl}
\hline $\mathrm{n}(\%)$ & Intoxicación & No intoxicación & Total \\
\hline Sospecha & $28(41,8)$ & $98(29,2)$ & $126(31,3)$ \\
No sospecha & $39(58,2)$ & $238(70,8)$ & $277(68,7)$ \\
Total & $67(100)$ & $336(100)$ & $403(100)$ \\
\hline
\end{tabular}

Sin significación estadística 
TABLA IV

\begin{tabular}{lccc}
\multicolumn{4}{c}{ TABLA IV } \\
\multicolumn{4}{c}{ RELACIÓ N ENTRE SO SPECHA DE INTO XICACIÓ N } \\
DIGITÁLICA Y PRO CEDEN CIA DE LA PETICIÓ N \\
\hline $\mathrm{n}(\%)$ & Ambulatorio & Hospitalario & Total \\
\hline Sospecha & $9(11,0)$ & $117(36,4)$ & $126(31,3)$ \\
No sospecha & $73(89,0)$ & $204(63,4)$ & $277(68,7)$ \\
Total & $82(20,3)$ & $321(79,7)$ & $403(100)$ \\
\hline
\end{tabular}

$p=0,0001$

\section{TABLA V}

RELACIÓ N ENTRE SO SPECHA DE INTO XICACIÓ N DIGITÁLICA Y FÁRM ACOS HIPO KALEM IANTES ASO CIADO S*

\begin{tabular}{lcll}
\hline n $(\%)$ & Con fármacos & \multicolumn{3}{l}{ Sin fármacos } & Total \\
\hline Sospecha & $31(22,8)$ & $103(36,1)$ & $134(31,8)$ \\
No sospecha & $105(77,2)$ & $182(63,9)$ & $287(68,2)$ \\
Total & $136(100)$ & $285(100)$ & $421(100)$ \\
\hline
\end{tabular}

* furosemida, diuréticos tiazídicos $p<0,005$

\section{TABLA VI}

RELACIÓN ENTRE SO SPECHA DE INTOXICACIÓN DIGITÁLICA Y FÁRM ACOS Q UE ELEVAN LA DIGO XINEM IA*

\begin{tabular}{llll}
\hline $\mathrm{n}(\%)$ & Con fármacos & \multicolumn{1}{l}{ Sin fármacos } & Total \\
\hline sospecha $(\%)$ & $16(21,3)^{* *}$ & $103(36,1)$ & $119(33,1)$ \\
no sospecha(\%) & $59(78,7)$ & $182(63,9)$ & $241(66,9)$ \\
total $(\%)$ & $75(100)$ & $285(100)$ & $360(100)$ \\
\hline
\end{tabular}

* quinidina, amiodarona, espironolactona, antagonistas del calcio. $* * p=0,01$

\section{DISCUSIÓN}

La OMS califica a los estudios de utilización de medicamentos como un instrumento valioso para conocer las consecuencias médicas, sociales o económicas derivadas del uso de los fármacos (15). Su finalidad no es valorar la eficacia o la seguridad de los fármacos prescritos (como ocurre, por ejemplo, en los ensayos clínicos y en los estudios de farmacovigilancia), sino la forma como se utilizan. Por tanto, el método empleado en nuestro estudio es válido para conseguir los objetivos propuestos: discriminar las intoxicaciones digitálicas que pasan inadvertidas clínicamente y las sospechadas que no se confirman analíticamente.

La toxicidad digitálica sigue siendo un tema de actualidad, a pesar que el uso de la digoxina tiende a disminuir y se conocen mejor los factores que alteran su farmacocinética. La intoxicación digitálica sigue siendo frecuente y es una de las reacciones adversas a medicamentos más prevalente $(1,11,16,17)$. En algunos estudios se estima que representa el $0,07 \%$ de todos los ingresos hospitalarios (18).

En nuestro trabajo, la distribución de las solicitudes de digoxinemia según la edad y el sexo, es similar a la de otros estudios recientes $(1,11,16,17)$, lo que sugiere indirectamente un hábito de uso (prescripción) similar por encima de las diferencias entre las poblaciones incluidas.

La proporción de intoxicación digitálica obtenida en el presente trabajo resulta acorde con la de otros autores, que varía entre 5-23\% según las características del estudio y de la población $(1,2,11,16,17)$. De todas ellas, la obtenida por Steiner et al. es la más parecida a la nuestra; esto se explica porque dicho autor también se basó en el análisis de las solicitudes de digoxinemia.

Las pocas solicitudes que cumplían los criterios de sospecha de intoxicación digitálica, y el que la mayoría de ellas presentaba un solo síntoma o signo de dicha toxicidad, lleva a pensar que en la práctica diaria la sospecha de intoxicación digitálica es pequeña o está poco fundamentada clínicamente, y que la digoxinemia se solicita por otras razones (ajuste de dosis, cumplimiento, etc.).

El papel de la edad en la frecuencia de la intoxicación digitálica no está tan claro como se pensaba. Hay autores que siguen defendiendo su influencia favorecedora (17). Otros encuentran diferencias significativas en dicha frecuencia en los grupos de mayor edad, pero sin significación estadística (1). Finalmente, hay estudios coincidiendo con nuestros resultados que demuestran la independencia del factor edad con la frecuencia de intoxicación digitálica (16). Es posible que estas opiniones dispares se deban a las diferencias metodológicas y poblacionales de los estudios realizados, así como al cambio en los hábitos de prescripción (reajuste de la dosificación de digoxina según la edad).

Las pocas sospechas de intoxicación confirmadas analíticamete y de intoxicaciones sospechadas en pacientes con digoxinemia superior a $2 \mathrm{ng} / \mathrm{mL}$, se pueden explicar por dos razones: no estar bien establecido el límite terapéutico superior de la digoxina (variable según paciente y/o situación clínica) por la existencia de solapamiento de síntomas y signos, y por la inespecificidad de los síntomas de esta intoxicación $(17,19)$. La imprecisión de este límite muestra la necesidad de estudios puntuales sobre este problema, cotidiano en la práctica clínica.

Aunque la frecuencia de intoxicación digitálica confirmadas analíticamente fue similar en los pacientes ambulatorios y en los hospitalizados, las sospechas fueron significativamente mayores en estos últimos. Esto puede relacionarse con la finalidad de la solicitud de digoxinemia en ambos grupos de enfermos: ajuste de dosis en los ambulatorios (normalmente estables clínicamente) y diagnóstico de intoxicación en los hospitalizados (menos estables clínicamente).

Esa misma razón (finalidad de la petición de digoxinemia) podría justificar que las sospecha de intoxicación fueran menores en los pacientes que se asociaban fármacos hipokalemiantes o que aumentan la concentración de digoxina. En ellos la petición de digoxinemia suele hacerse por el mero hecho de recibir tales fármacos, al margen de la sintomatología que presenten. Por otro lado, que el número de intoxicaciones confirmadas fuese similar en los pacientes que recibían uno $\mathrm{u}$ otro tipo de fármacos (hipokalemiantes o aumentadores de la digoxinemia), apoya el razonamiento anterior (20).

En la mayoría de las ocasiones las intoxicaciones digitálicas (>2 ng/mL) no se sospechan, ni las sospechas de intoxicación se confirman. Se puede concluir que la sospecha de esta intoxicación no suele relacionarse con los niveles plasmáticos de digoxina y, por tanto, que su límite tóxico es impreciso. 


\section{Bibliografía}

1. Marik PE, From L. A cases series of hospitalized patients with elevated digoxin level. Am J Med 1998; 105: 110-115.

2. Steiner JF, Robbins LS, Hammermeister KE, Roth SC, Hammond WS. Incidence of digoxin toxicity in outpatients. West J Med 1994; 161: 474-478.

3. Lewis RP. Clinical use of serum digoxin concentrations. Am J Cardiol 1992; 69: 97G-107G

4. Wofford JL, Ettinger WH. Risk factors and manifestations of digoxin toxicity in the elderly. Am J Emerg Med 1991; 9 (Supl. 1): 11-15.

5. van Asselt DZ, Collas D, Hoefnagels WH, Rai GS. Digoxin poisoning in patients of 2 geriatric departments in London: prevalence and mortality. Tijdschr Gerontol Geriatr 1993; 24: 150-155.

6. Ordog GJ, Benaron S, Bhasin V, Wasserberger J, Balasubramanium S. Serum digoxin levels and mortality in 5,100 patients. Ann Emerg Med 1987; 16: 32-39.

7. Wolin MJ. Digoxin visual toxicity with therapeutic blood levels of digoxin. Am J Ophthalmol 1998; 125: 406-407.

8. Kelly RA, Smith TW. Recognition and management of digitalis toxicity. Am J Cardiol 1992; 69: 108G-118G.

9. Wofford JL, Hickey AR, Ettinger WH, Furberg CD. Lack of age-related differences in the clinical presentation of digoxin toxicity. Arch Intern Med 1992; 152: 2261-2264.

10. Gosselink AT, van Veldhhuisen DJ, Crijns HJ. When, and when not, to use dogixin in the elderly. Drug Aging 1997; 10: 411-420.

11. Abad-Santos F, Carcas AJ, Ibáñez C, Frías J. Digoxin level and clinical manifestations as determinants in the diagnosis of digoxin toxicity. Ther Drug Monit 2000; 22: 163-168.
12. Tuncok Y, Hanzan E, Oto O, Guven H, Catalyurek H, Kalkan S. Relationship between high serum digoxin levels and toxicity. Int $\mathrm{J}$ Clin Pharmacol Ther 1997; 35: 366-368.

13. Sánchez de la Rosa R, Rodríguez Hernández N, Cueto Guerreiro T, Rebollo Velazquez A, de Armas Alonso A, Sánchez de la Rosa E. Epidemilogical surveillance of patients treated with digoxin. Arch Inst Cardiol Mex 1996; 66: 510-518.

14. Canas F, Tanasijevic MJ, Ma'luf N, Bates DW. Evaluating the appropriateness of digoxin level monitoring. Arch Intern Med 1999; 159. 363-368.

15. Comité de Expertos de la OMS. Serie de Informes Técnicos nº 615. OMS, Ginebra, 1977.

16. Rich MW, McSherry F, Williford WO, Yusuf S (Digitalis Investigation Group). Effect of age on mortality hospitalizations and response to digoxin in patients with heart failure: the DIG study. J Am Coll Cardiol 2001; 38: 806-813.

17. MiuraT, Kojima R, Sugiura Y, Mizutani M, Takatsu F, Suzuki Y. Effect of aging on the incidence of digoxin toxicity. Ann Pharmacother 2000, 34: 427-432.

18. Gandhi AJ, Vlasses PH, Morton DJ, Bauman JL. Economic impact of digoxin toxicity. Pharmacoeconomics 1997; 12: 175-181.

19. Sinkiewicz W, Marzec A, Jankowski A, Hoffmann A. Digoxin level as limited drug toxicity indicator in the assessment of post-digitalis arrhythmia. Wiad Lek 1994: 47: 808-812.

20. Mordel A, Halkin H, Zulty L, Almog S, Ezra D. Quinidine enhances digitalis toxicity at therapeutic serum digoxin levels. Clin Pharmacol Ther 1993; 53: 457-462. 